\title{
Inverse inhomogeneous penetrable obstacle scattering problems in a stratified medium
}

\author{
Guoping Zhan ${ }^{1}$ and Lihan Liu²*
}

\section{"Correspondence:}

liulihan@mail2.sysu.edu.cn

2Department of Mathematics,

Chongqing Normal University,

Chongqing, 401331, China

Full list of author information is

available at the end of the article (c) The Author(s) 2017. This article is distributed under the terms of the Creative Commons Attribution 4.0 International License (http://creativecommons.org/licenses/by/4.0/), which permits unrestricted use, distribution, and reproduction in any medium, provided you give appropriate credit to the original author(s) and the source, provide a link to the Creative Commons license, and indicate if changes were made.

\begin{abstract}
In this paper we study the inverse inhomogeneous penetrable obstacle scattering problems in a stratified medium. On the basis of the uniqueness and existence of solutions for the direct scattering by an inhomogeneous penetrable obstacle in a stratified medium, we first establish an a priori estimate of the solution on some part of the penetrable interfaces $S_{i}(i=1,2)$, which plays an important role in the inverse scattering problems, and then we prove that both the penetrable interfaces $S_{i}(i=1,2)$ and the refractive index $n(x)$ of the inhomogeneous penetrable obstacle $\Omega_{2}$ can be uniquely determined from knowledge of the far-field pattern $u^{\infty}(\widehat{x}, d)$ $\left(\widehat{x}, d \in \mathbb{S}, \mathbb{S}\right.$ is the unit sphere of $\left.\mathbb{R}^{3}\right)$ for an incident plane wave $u^{i}(x)=e^{i k_{1} x \cdot d}\left(x \in \mathbb{R}^{3}\right)$.
\end{abstract}

Keywords: inverse scattering problems; inhomogeneous penetrable obstacle; stratified medium; Helmholtz equation

\section{Introduction}

In this paper, we study the inverse inhomogeneous penetrable obstacle scattering problems in a stratified medium. In many branches of science and engineering such as radar and sonar, remote sensing, geophysics, geological exploration, nondestructive testing and medical imaging, the background medium may be described as a stratified medium rather than a homogeneous medium. Consequently, one possible model would be an inhomogeneous penetrable obstacle buried in a stratified medium. For simplicity and without loss of generality, in this paper we consider the case where the inhomogeneous penetrable obstacle is buried in a stratified medium with two layers. To be specific, let $\Omega_{3} \subset \mathbb{R}^{3}$ be a bounded homogeneous medium with a closed $C^{2}$ boundary surface $S_{2}$ such that $\mathbb{R}^{3} \backslash \overline{\Omega_{3}}$ is divided into two connected domains $\Omega_{2}$ and $\Omega_{1}$ by a closed $C^{2}$ boundary surface $S_{1}$, where $\Omega_{2}$ is an inhomogeneous penetrable obstacle and $\Omega_{1}$ is an unbounded homogeneous medium.

The problems of scattering by an inhomogeneous penetrable obstacle in a stratified medium with two layers in $\mathbb{R}^{3}$ can be described as the following Helmholtz equations with transmission boundary conditions on their interfaces and Sommerfeld radiation condition, that is, the following boundary value problem:

$$
\Delta u(x)+k_{1}^{2} u(x)=0, \quad \text { in } \Omega_{1},
$$




$$
\begin{aligned}
& \Delta v(x)+k_{2}^{2} n(x) v(x)=0, \quad \text { in } \Omega_{2}, \\
& \Delta w(x)+k_{3}^{2} w(x)=0, \quad \text { in } \Omega_{3}, \\
& u(x)-v(x)=0, \quad \frac{\partial u(x)}{\partial v}-\lambda_{1} \frac{\partial v(x)}{\partial v}=0, \quad \text { on } S_{1}, \\
& v(x)-w(x)=0, \quad \frac{\partial v(x)}{\partial v}-\lambda_{2} \frac{\partial w(x)}{\partial v}=0, \quad \text { on } S_{2}, \\
& \lim _{r \rightarrow \infty} r\left[\frac{\partial u^{s}(x)}{\partial r}-i k_{1} u^{s}(x)\right]=0,
\end{aligned}
$$

where $r=|x|=\sqrt{x_{1}^{2}+x_{2}^{2}+z^{2}}, x=\left(x_{1}, x_{2}, z\right) \in \mathbb{R}^{3}, \Delta=\frac{\partial^{2}}{\partial x_{1}^{2}}+\frac{\partial^{2}}{\partial x_{2}^{2}}+\frac{\partial^{2}}{\partial z^{2}}$ is the Laplacian, $u(x)=u\left(x_{1}, x_{2}, z\right), v(x)=v\left(x_{1}, x_{2}, z\right)$ and $w(x)=w\left(x_{1}, x_{2}, z\right)$ are the time-harmonic velocity potentials, $v$ is the unit outward normal to the penetrable interface $S_{i}(i=1,2)$ and $n(x)=n\left(x_{1}, x_{2}, z\right) \in C^{0, \alpha}\left(\overline{\Omega_{2}}\right), 0<\alpha<1$, is the refractive index of an inhomogeneous penetrable obstacle with $\mathfrak{R}[n(x)]>0, \mathfrak{\Im}[n(x)] \geq 0$. Here, the total field $u(x)=u^{i}(x)+u^{s}(x)$ is given as the sum of the unknown scattered wave $u^{s}(x)=u^{s}\left(x_{1}, x_{2}, z\right)$ which is required to satisfy the Sommerfeld radiation condition (1.6) and the incident wave $u^{i}(x)=u^{i}\left(x_{1}, x_{2}, z\right)$, $k_{j}>0(j=1,2,3)$ is the wave number given by $k_{j}=\frac{\omega_{j}}{c_{j}}(j=1,2,3)$ in terms of the frequency $\omega_{j}(j=1,2,3)$ and the wave speed $c_{j}(j=1,2,3)$ in the corresponding medium $\Omega_{j}(j=1,2,3)$. The distinct wave numbers $k_{j}(j=1,2,3)$ correspond to the fact that the medium consists of several physically different materials. On the penetrable interfaces $S_{i}(i=1,2)$, the so-called transmission conditions (1.4)-(1.5) with two constants $\lambda_{1}>0$ and $\lambda_{2}>0$ are imposed, respectively, which represent the continuity of the medium and equilibrium of the forces acting on them.

For the uniqueness results for inverse scattering by an inhomogeneity with compact support in a homogeneous medium, see Hähner [1], Nachman [2], Novikov [3], and Ramm $[4,5]$, or see Colton and Kress [6], Isakov [7], and Kirsch [8] for a comprehensive discussion. In the case when the obstacle is impenetrable, Liu et al. have proved the unique determination of some inverse scattering problems, see $[9,10]$, or see Hähner [11], and when the obstacle is penetrable, some results concerned with the unique determination of the inverse scattering problems can be found in Athanasiadis and Stratis [12], Kirsch and Päivärinta [13], Liu and Zhang [14], Nachman, Päivärinta and Teirlilä [15], and Yan [16]. Moreover, Giorgi, Brignone, Aramini, and Piana [17] have presented a hybrid approach, which merges a qualitative and a quantitative method to optimize the way of exploiting the $a$ priori information on the background within the inversion procedure, to numerically solving two-dimensional electromagnetic inverse scattering problems, whereby the unknown scatterer is hosted by a possibly inhomogeneous background.

The rest of the paper is organized as follows: in Section 2, we recall the uniqueness and existence of solutions for the direct scattering by an inhomogeneous obstacle in a stratified medium, which will be useful in the rest of the paper. In Section 3, we will establish a priori estimate of the solution on some part of the penetrable interfaces $S_{i}(i=1,2)$, which plays an important role in the inverse scattering problem. In Section 4, we will prove that both the penetrable interfaces $S_{i}(i=1,2)$ and the refractive index $n(x)$ of the inhomogeneous penetrable obstacle $\Omega_{2}$ can be uniquely determined from knowledge of the far-field pattern $u^{\infty}(\widehat{x}, d)\left(\widehat{x}, d \in \mathbb{S}, \mathbb{S}\right.$ is the unit sphere of $\left.\mathbb{R}^{3}\right)$ for an incident plane wave $u^{i}(x)=e^{i k_{1} x \cdot d}$. 


\section{Preliminaries}

In this section, we recall the uniqueness and existence of solutions for the direct scattering by an inhomogeneous obstacle in a stratified medium, which we have addressed in [18]. These results will be useful in the rest of the paper. From now on, we assume that $k_{1}, k_{2}, k_{3}, \lambda_{1}, \lambda_{2}$ are given positive numbers and that $k_{2}^{2}$ is not a Neumann eigenvalue of $\Delta v(x)+k_{2}^{2} n(x) v(x)=0$ in $\Omega_{2}$.

The incident wave fields $u^{i}(x)=u^{i}\left(x_{1}, x_{2}, z\right)$ may be an incident plane wave $e^{i k_{1} x \cdot d}$ or point source $\Phi_{j}\left(\cdot, z_{j}\right)(j=1,3)$, which will be given below, where $d \in \mathbb{S}$ is the incident direction, $z_{j} \in \Omega_{j}(j=1,3)$. Denote by $u^{s}(\cdot, d)$ the scattered field for an incident plane wave $u^{i}(\cdot, d)$ and by $u^{\infty}(\cdot, d)$ the corresponding far-field pattern, and denote by $u^{s}\left(\cdot, z_{j}\right)(j=1,3)$ the scattered field for an incident point source $\Phi_{j}\left(\cdot, z_{j}\right)(j=1,3)$ and by $\Phi^{\infty}\left(\cdot, z_{j}\right)(j=1,3)$ the corresponding far-field pattern.

We will look for the solution $u(x) \in C^{2}\left(\Omega_{1}\right) \cap C^{1, \alpha}\left(\overline{\Omega_{1}}\right), v(x) \in C^{2}\left(\Omega_{2}\right) \cap C^{1, \alpha}\left(\overline{\Omega_{2}}\right)$ and $w(x) \in C^{2}\left(\Omega_{3}\right) \cap C^{1, \alpha}\left(\overline{\Omega_{3}}\right)$ satisfying the following Helmholtz equations with transmission boundary conditions on their interfaces and Sommerfeld radiation condition, that is, the following boundary value problem:

$$
\begin{aligned}
& \Delta u(x)+k_{1}^{2} u(x)=0, \quad \text { in } \Omega_{1}, \\
& \Delta v(x)+k_{2}^{2} n(x) v(x)=0, \quad \text { in } \Omega_{2}, \\
& \Delta w(x)+k_{3}^{2} w(x)=0, \quad \text { in } \Omega_{3}, \\
& u(x)-v(x)=f(x), \quad \frac{\partial u(x)}{\partial v}-\lambda_{1} \frac{\partial v(x)}{\partial v}=g(x), \quad \text { on } S_{1}, \\
& v(x)-w(x)=p(x), \quad \frac{\partial v(x)}{\partial v}-\lambda_{2} \frac{\partial w(x)}{\partial v}=q(x), \quad \text { on } S_{2}, \\
& \lim _{r \rightarrow \infty} r\left[\frac{\partial u^{s}(x)}{\partial r}-i k_{1} u^{s}(x)\right]=0,
\end{aligned}
$$

where $f(x) \in C^{1, \alpha}\left(S_{1}\right), g(x) \in C^{0, \alpha}\left(S_{1}\right), p(x) \in C^{1, \alpha}\left(S_{2}\right)$ and $q(x) \in C^{0, \alpha}\left(S_{2}\right)$ are given functions from Hölder spaces with $0<\alpha<1$. For the scattering problem, if the incident field $u^{i}(x)=u^{i}\left(x_{1}, x_{2}, z\right)$ is the incident plane wave $e^{i k_{1} x \cdot d}$ or the point source $\Phi_{1}\left(\cdot, z_{1}\right)$ with $z_{1} \in \Omega_{1}$, then $f(x)=-u^{i}(x), g(x)=-\frac{\partial u^{i}(x)}{\partial v}, p=0, q=0$, and if the incident field $u^{i}(x)=u^{i}\left(x_{1}, x_{2}, z\right)$ is the point source $\Phi_{3}\left(\cdot, z_{3}\right)$ with $z_{3} \in \Omega_{3}$, then $f(x)=u^{i}(x), g(x)=$ $\lambda_{1} \lambda_{2} \frac{\partial u^{i}(x)}{\partial v}, p(x)=-u^{i}(x), q(x)=-\lambda_{2} \frac{\partial u^{i}(x)}{\partial v}$.

So we can recall the following three lemmas.

Lemma 1 There exists at most one solution for the Helmholtz equations (2.1)-(2.3) with transmission boundary conditions (2.4)-(2.5) and Sommerfeld radiation condition (2.6), that is, the boundary value problem (2.1)-(2.6).

Proof The proof is analogous to the proof of Theorem 1 in [18] and, hence, is omitted.

Lemma 2 There exists a unique solution for the Helmholtz equations (2.1)-(2.3) with transmission boundary conditions (2.4)-(2.5) and Sommerfeld radiation condition (2.6), that is, 
the boundary value problem (2.1)-(2.6). In particular, such a solution satisfies the estimate

$$
\begin{aligned}
& \|u(x)\|_{1, \alpha, \overline{\Omega_{1}}}+\|v(x)\|_{1, \alpha, \overline{\Omega_{2}}}+\|w(x)\|_{1, \alpha, \overline{\Omega_{3}}} \\
& \quad \leq C\left(\|f(x)\|_{1, \alpha, S_{1}}+\|g(x)\|_{0, \alpha, S_{1}}+\|p(x)\|_{1, \alpha, S_{2}}+\|q(x)\|_{0, \alpha, S_{2}}\right),
\end{aligned}
$$

for some positive constant $C=C(\alpha)$.

Proof The proof is analogous to the proof of Theorem 2 in [18] and, hence, is omitted.

Lemma 3 For the scattering of the incident plane wave $u^{i}(\cdot, d)$ with the incident direction $d \in \mathbb{S}$ and the incident point source $\Phi(\cdot, z)$ from the inhomogeneous penetrable obstacle $\Omega_{2}$, we have

$$
\Phi^{\infty}(\widehat{x}, z)= \begin{cases}\frac{1}{4 \pi} u^{s}(z,-\widehat{x}), & z \in \Omega_{1}, \\ \frac{\lambda_{1} \lambda_{2}}{4 \pi} w(z,-\widehat{x}), & z \in \Omega_{3},\end{cases}
$$

where $\widehat{x}=\frac{x}{|x|} \in \mathbb{S}$ is the observation direction.

Proof The proof is analogous to the proof of Theorem 3 in [18] and, hence, is omitted.

\section{Results and discussion}

In this section, we will establish an a priori estimate of the solution on the penetrable interfaces $S_{i}(i=1,2)$, which plays an important role in the inverse scattering problem.

To prove the next two lemmas, we first need the fundamental solution $\Phi_{j}(j=1,2,3)$ to the Helmholtz equation with wave number $k_{j}(j=1,2,3)$ given by

$$
\Phi_{j}(x, y)=\frac{e^{i k_{j}|x-y|}}{4 \pi|x-y|}, \quad x, y \in \mathbb{R}^{3}, x \neq y, j=1,2,3 .
$$

Define the single-layer and double-layer potentials $\widetilde{S}_{i, j}(i=1,2, j=1,2,3)$ and $\widetilde{K}_{i, j}(i=1,2, j=$ $1,2,3)$, respectively, by

$$
\begin{aligned}
& \left(\widetilde{S}_{i, j} \phi\right)(x) \triangleq \int_{S_{i}} \Phi_{j}(x, y) \phi(y) d s(y), \quad x \in \mathbb{R}^{3} \backslash S_{i}, i=1,2, j=1,2,3, \\
& \left(\widetilde{K}_{i, j} \phi\right)(x) \triangleq \int_{S_{i}} \frac{\partial \Phi_{j}(x, y)}{\partial v(y)} \phi(y) d s(y), \quad x \in \mathbb{R}^{3} \backslash S_{i}, i=1,2, j=1,2,3,
\end{aligned}
$$

and the normal derivative operators $\widetilde{K}_{i, j}^{\prime}(i=1,2, j=1,2,3)$ and $\widetilde{T}_{i, j}(i=1,2, j=1,2,3)$ by

$$
\begin{aligned}
& \left(\widetilde{K}_{i, j}^{\prime} \phi\right)(x) \triangleq \frac{\partial}{\partial v(x)} \int_{S_{i}} \Phi_{j}(x, y) \phi(y) d s(y), \quad x \in \mathbb{R}^{3} \backslash S_{i}, i=1,2, j=1,2,3, \\
& \left(\widetilde{T}_{i, j} \phi\right)(x) \triangleq \frac{\partial}{\partial \nu(x)} \int_{S_{i}} \frac{\partial \Phi_{j}(x, y)}{\partial \nu(y)} \phi(y) d s(y), \quad x \in \mathbb{R}^{3} \backslash S_{i}, i=1,2, j=1,2,3 .
\end{aligned}
$$

These operators restricted on the penetrable interfaces $S_{i}(i=1,2)$ will be denoted by $S_{i, j}$, $K_{i, j}, K_{i, j}^{\prime}$ and $T_{i, j}(i=1,2, j=1,2,3)$, respectively. For the proof of the next two lemmas, we 
also need the volume potential

$$
(V \phi)(x) \triangleq k_{2}^{2} \int_{\Omega_{2}} \Phi_{2}(x, y)[n(y)-1] \phi(y) d y, \quad x \in \mathbb{R}^{3},
$$

and its normal derivative operator denoted by $V^{\prime}$. For mapping properties of these operators in the classical spaces of continuous and Hölder continuous functions, see the monographs of Colton and Kress [6, 19].

Let $\Omega$ be the complement of $\Omega_{1}$, that is, $\Omega \triangleq \mathbb{R}^{3} \backslash \overline{\Omega_{1}}$. Choose a large ball $B_{R}$ centered at the origin such that $\bar{\Omega} \subset B_{R}$ and let $\Omega_{R}=B_{R} \backslash \bar{\Omega}$. Denote by $D$ any of $\overline{\Omega_{R}}, \overline{\Omega_{2}}, \overline{\Omega_{3}}, S_{1}$ or $S_{2}$. Let $x^{\prime} \in D$ be an arbitrarily fixed point and denote by the weight space $C_{0}(D)$ consisting of all continuous functions $h(x) \in C\left(D \backslash\left\{x^{\prime}\right\}\right)$, such that

$$
\lim _{x \rightarrow x^{\prime}}\left|\left(x-x^{\prime}\right) h(x)\right|
$$

exists. It can easily be verified that $C_{0}(D)$ is a Banach space equipped with the weighted maximum norm

$$
\|h(x)\|_{0, D} \triangleq \sup _{x \neq x^{\prime}, x \in D}\left|\left(x-x^{\prime}\right) h(x)\right|
$$

Let $B_{1}, B_{2}$ be two small balls with center $x^{(1)}$ and radii $r_{1}, r_{2}$, respectively, satisfying that $x^{(1)} \in S_{1}, r_{1}<r_{2}, B_{2} \cap \overline{\Omega_{3}}=\emptyset$. Now we consider the scattering problem of the incident point source $\Phi_{1}\left(x, z_{1}\right)$ with $z_{1} \in B_{1} \cap \Omega_{1}$. For the proof of the unique determination of the boundary interface $S_{1}$ in the inverse scattering problem in the next section, we first study the behavior of the solution $v$ on some part of the boundary interface $S_{1}$.

Lemma 4 Let $u(x) \in C^{2}\left(\Omega_{1}\right) \cap C^{1, \alpha}\left(\overline{\Omega_{1}}\right), v(x) \in C^{2}\left(\Omega_{2}\right) \cap C^{1, \alpha}\left(\overline{\Omega_{2}}\right)$ and $w(x) \in C^{2}\left(\Omega_{3}\right) \cap$ $C^{1, \alpha}\left(\overline{\Omega_{3}}\right)$ be a solution of the boundary value problem (2.1)-(2.6) with $f(x), g(x), p(x), q(x)$ given below, then there exists a constant $C>0$ such that

$$
\begin{aligned}
\| v(x) & \left\|_{\infty, S_{1} \backslash B_{2}}+\right\| \frac{\partial v(x)}{\partial v} \|_{\infty, S_{1} \backslash B_{2}} \\
\leq & C\left(\left\|f_{1}(x)\right\|_{0, S_{1}}+\left\|g_{1}(x)\right\|_{0, S_{1}}+\left\|f_{1}(x)\right\|_{1, \alpha, S_{1} \backslash B_{1}}\right. \\
& +\left\|g_{1}(x)\right\|_{0, \alpha, S_{1} \backslash B_{1}}+\left\|p_{1}(x)\right\|_{1, \alpha, S_{2}}+\left\|q_{1}(x)\right\|_{0, \alpha, S_{2}} \\
& \left.+\left\|\Phi_{2}\left(x, z_{1}\right)\right\|_{L^{2}\left(\Omega_{2}\right)}\right)
\end{aligned}
$$

where $f(x)=f_{1}(x)=\frac{1}{\lambda_{1}} \Phi_{2}\left(x, z_{1}\right)-\Phi_{1}\left(x, z_{1}\right), g(x)=g_{1}(x)=\frac{\partial \Phi_{2}\left(x, z_{1}\right)}{\partial v}-\frac{\partial \Phi_{1}\left(x, z_{1}\right)}{\partial v}, p(x)=p_{1}(x)=$ $-\frac{1}{\lambda_{1}} \Phi_{2}\left(x, z_{1}\right), q(x)=q_{1}(x)=-\frac{\partial \Phi_{2}\left(x, z_{1}\right)}{\partial v}$ and $z_{1} \in B_{1} \cap \Omega_{1}$.

Proof From [19] and [6], we look for the unique solution in the form

$$
\begin{aligned}
& u(x)=\lambda_{1}\left(\widetilde{K}_{1,1} \varphi_{1}\right)(x)+\left(\widetilde{S}_{1,1} \phi_{1}\right)(x), \quad \text { in } \Omega_{1}, \\
& v(x)=\left(\widetilde{K}_{1,2} \varphi_{1}\right)(x)+\left(\widetilde{S}_{1,2} \phi_{1}\right)(x)+\lambda_{2}\left(\widetilde{K}_{2,2} \varphi_{2}\right)(x)+\left(\widetilde{S}_{2,2} \phi_{2}\right)(x)+(V v)(x), \quad \text { in } \Omega_{2}, \\
& w(x)=\left(\widetilde{K}_{2,3} \varphi_{2}\right)(x)+\left(\widetilde{S}_{2,3} \phi_{2}\right)(x), \quad \text { in } \Omega_{3},
\end{aligned}
$$


with four densities $\varphi_{1}(x) \in C^{1, \alpha}\left(S_{1}\right), \phi_{1}(x) \in C^{0, \alpha}\left(S_{1}\right), \varphi_{2}(x) \in C^{1, \alpha}\left(S_{2}\right), \phi_{2}(x) \in C^{0, \alpha}\left(S_{2}\right)$. Then, from the transmission boundary conditions (2.4)-(2.5), we can see that the velocity potentials $u(x), v(x)$ and $w(x)$ given by (3.10)-(3.12) solve the boundary value problem (2.1)-(2.6) if the four mentioned densities satisfy the following system of integral equations:

$$
\begin{aligned}
& \quad \frac{\lambda_{1}+1}{2} \varphi_{1}(x)+\left[\left(\lambda_{1} K_{1,1}-K_{1,2}\right) \varphi_{1}\right](x)+\left[\left(S_{1,1}-S_{1,2}\right) \phi_{1}\right](x)-\left(\lambda_{2} K_{2,2} \varphi_{2}+S_{2,2} \phi_{2}\right)(x) \\
& \quad-(V v)(x)=f_{1}(x), \quad \text { on } S_{1}, \\
& -\frac{\lambda_{1}+1}{2} \phi_{1}(x)+\lambda_{1}\left[\left(T_{1,1}-T_{1,2}\right) \varphi_{1}\right](x)+\left[\left(K_{1,1}^{\prime}-\lambda_{1} K_{1,2}^{\prime}\right) \phi_{1}\right](x) \\
& \quad-\lambda_{1}\left(\lambda_{2} T_{2,2} \varphi_{2}+K_{2,2}^{\prime} \phi_{2}\right)(x)-\lambda_{1}\left(V^{\prime} v\right)(x)=g_{1}(x), \quad \text { on } S_{1}, \\
& \frac{\lambda_{2}+1}{2} \varphi_{2}(x)+\left[\left(\lambda_{2} K_{2,2}-K_{2,3}\right) \varphi_{2}\right](x)+\left[\left(S_{2,2}-S_{2,3}\right) \phi_{2}\right](x)+\left(K_{1,2} \varphi_{1}+S_{1,2} \phi_{1}\right)(x) \\
& \quad+(V v)(x)=p_{1}(x), \quad \text { on } S_{2}, \\
& -\frac{\lambda_{2}+1}{2} \phi_{2}(x)+\lambda_{2}\left[\left(T_{2,2}-T_{2,3}\right) \varphi_{2}\right](x)+\left[\left(K_{2,2}^{\prime}-\lambda_{2} K_{2,3}^{\prime}\right) \phi_{2}\right](x) \\
& \quad+\left(T_{1,2} \varphi_{1}+K_{1,2}^{\prime} \phi_{1}\right)(x)+\lambda_{2}\left(V^{\prime} v\right)(x)=q_{1}(x), \quad \text { on } S_{2} .
\end{aligned}
$$

From the integral equations (3.13) and (3.15) and by using Theorem 3.4 in [6], we can see that $\varphi_{1}(x) \in C^{1, \alpha}\left(S_{1}\right)$ and $\varphi_{2}(x) \in C^{1, \alpha}\left(S_{2}\right)$. Define the product space

$$
X \triangleq C^{1, \alpha}\left(\overline{\Omega_{1}}\right) \times C^{1, \alpha}\left(\overline{\Omega_{2}}\right) \times C^{1, \alpha}\left(\overline{\Omega_{3}}\right) \times C^{1, \alpha}\left(S_{1}\right) \times C^{0, \alpha}\left(S_{1}\right) \times C^{1, \alpha}\left(S_{2}\right) \times C^{0, \alpha}\left(S_{2}\right),
$$

then $X$ can be chosen as the solution space of the above system (3.10)-(3.16). Assume that the operator $A: X \rightarrow X$ is given in the following matrix form:

$$
A=\left(\begin{array}{ccccccc}
0 & 0 & 0 & -\lambda_{1} \widetilde{K}_{1,1} & -\widetilde{S}_{1,1} & 0 & 0 \\
0 & -V & 0 & -\widetilde{K}_{1,2} & -\widetilde{S}_{1,2} & -\lambda_{2} \widetilde{K}_{2,2} & -\widetilde{S}_{2,2} \\
0 & 0 & 0 & 0 & 0 & -\widetilde{K}_{2,3} & -\widetilde{S}_{2,3} \\
0 & -\frac{2 V}{\lambda_{1}+1} & 0 & \frac{2\left(\lambda_{1} K_{1,1}-K_{1,2}\right)}{\lambda_{1}+1} & \frac{2\left(S_{1,1}-S_{1,2}\right)}{\lambda_{1}+1} & -\frac{2 \lambda_{2} K_{2,2}}{\lambda_{1}+1} & -\frac{2 S_{2,2}}{\lambda_{1}+1} \\
0 & -\frac{2 \lambda_{1} V^{\prime}}{\lambda_{1}+1} & 0 & \frac{2 \lambda_{1}\left(T_{1,2}-T_{1,1}\right)}{\lambda_{1}+1} & \frac{2\left(\lambda_{1} K_{1,2}^{\prime}-K_{1,1}^{\prime}\right)}{\lambda_{1}+1} & \frac{2 \lambda_{1} \lambda_{2} T_{2,2}}{\lambda_{1}+1} & \frac{2 \lambda_{1} K_{2,2}^{\prime}}{\lambda_{1}+1} \\
0 & \frac{2 V}{\lambda_{2}+1} & 0 & \frac{2 K_{1,2}}{\lambda_{2}+1} & \frac{2 S_{1,2}}{\lambda_{2}+1} & -\frac{2\left(\lambda_{2} K_{2,2}-K_{2,3}\right)}{\lambda_{2}+1} & -\frac{2\left(S_{2,2}-S_{2,3}\right)}{\lambda_{2}+1} \\
0 & -\frac{2 \lambda_{2} V^{\prime}}{\lambda_{2}+1} & 0 & -\frac{2 T_{1,2}}{\lambda_{2}+1} & -\frac{2 K_{1,2}^{\prime}}{\lambda_{2}+1} & \frac{2 \lambda_{2}\left(T_{2,3}-T_{2,2}\right)}{\lambda_{2}+1} & \frac{2\left(\lambda_{2} K_{2,3}^{\prime}-K_{2,2}^{\prime}\right)}{\lambda_{2}+1}
\end{array}\right) .
$$

Hence, the above system (3.10)-(3.16) can be rewritten in the abbreviated form

$$
(I+A) U(x)=R(x)
$$

where $I$ is the identity operator, $U(x)=\left(u(x), v(x), w(x), \varphi_{1}(x), \phi_{1}(x), \varphi_{2}(x), \phi_{2}(x)\right)^{T}$, and

$$
R(x)=\left(0,0,0, \frac{2 f_{1}(x)}{\lambda_{1}+1},-\frac{2 g_{1}(x)}{\lambda_{1}+1}, \frac{2 p_{1}(x)}{\lambda_{2}+1},-\frac{2 q_{1}(x)}{\lambda_{2}+1}\right)^{T} .
$$


Also, we define another weighted product space

$$
Y \triangleq C_{0}\left(\overline{\Omega_{R}}\right) \times C^{1, \alpha}\left(\overline{\Omega_{2}}\right) \times C_{0}\left(\overline{\Omega_{3}}\right) \times C_{0}\left(S_{1}\right) \times C_{0}\left(S_{1}\right) \times C^{1, \alpha}\left(S_{2}\right) \times C^{0, \alpha}\left(S_{2}\right) .
$$

From [20] and [13], we know that all entries of the matrix operator $A$ are compact, hence, we can easily see that the matrix operator $A$ is compact in the weighted product space $Y$. By using Theorem 2 in [18], we know that the operator $I+A$ has a trivial null space in the weighted product space $X$. Consequently, by applying the Riesz-Fredholm theory to the dual system $\langle X, Y\rangle$ with the $L^{2}$ bilinear form, we can easily see that the adjoint operator $I+A^{\prime}$ has a trivial null space in the weighted product space $Y$. Then, by applying the Riesz-Fredholm theory again to the dual system $\langle Y, Y\rangle$ with the $L^{2}$ bilinear form, we can easily see that the operator $I+A$ has a trivial null space in the weighted product space $Y$. Hence, system (3.18) is uniquely solvable in the weighted product space $Y$, and the solution depends continuously on the right-hand side:

$$
\begin{aligned}
\|U(x)\|_{0} \triangleq & \|u(x)\|_{0, \overline{\Omega_{R}}}+\|v(x)\|_{1, \alpha, \overline{\Omega_{2}}}+\|w(x)\|_{0, \overline{\Omega_{3}}} \\
& +\left\|\varphi_{1}(x)\right\|_{0, S_{1}}+\left\|\phi_{1}(x)\right\|_{0, S_{1}}+\left\|\varphi_{2}(x)\right\|_{1, \alpha, S_{2}}+\left\|\phi_{2}(x)\right\|_{0, \alpha, S_{2}} \\
\leq & C\left(\left\|f_{1}(x)\right\|_{0, S_{1}}+\left\|g_{1}(x)\right\|_{0, S_{1}}+\left\|p_{1}(x)\right\|_{1, \alpha, S_{2}}+\left\|q_{1}(x)\right\|_{0, \alpha, S_{2}}\right. \\
& \left.+\left\|\Phi_{2}\left(x, z_{1}\right)\right\|_{L^{2}\left(\Omega_{2}\right)}\right) .
\end{aligned}
$$

In particular, this implies that

$$
\begin{aligned}
\|v(x)\|_{\infty, S_{1} \backslash B_{2}} \leq & C\left(\left\|f_{1}(x)\right\|_{0, S_{1}}+\left\|g_{1}(x)\right\|_{0, S_{1}}+\left\|p_{1}(x)\right\|_{1, \alpha, S_{2}}+\left\|q_{1}(x)\right\|_{1, \alpha, S_{2}}\right. \\
& \left.+\left\|\Phi_{2}\left(x, z_{1}\right)\right\|_{L^{2}\left(\Omega_{2}\right)}\right) .
\end{aligned}
$$

Let $B_{12}$ be a ball of radius $r_{12}$ and centered at $x^{(1)}$ with $r_{1}<r_{12}<r_{2}$ and assume that $\rho_{1}(x) \in$ $C^{2}\left(S_{1}\right)$ is a function satisfying $\rho_{1}(x)=0$ for $x \in S_{1} \backslash B_{2}$ and $\rho_{1}(x)=1$ in the neighborhood of $B_{12}, \rho_{2}(x) \in C^{2}\left(S_{1}\right)$ is another function satisfying $\rho_{2}(x)=0$ for $x \in S_{1} \backslash B_{12}$ and $\rho_{2}(x)=1$ in the neighborhood of $B_{1}$.

We rewrite $U(x)$ in the form

$$
U(x)=\left(\begin{array}{c}
u(x) \\
v(x) \\
w(x) \\
\varphi_{1}(x) \\
\phi_{1}(x) \\
\varphi_{2}(x) \\
\phi_{2}(x)
\end{array}\right)=\left(\begin{array}{c}
\rho_{1}(x) u(x) \\
\rho_{1}(x) v(x) \\
w(x) \\
\rho_{1}(x) \varphi_{1}(x) \\
\rho_{1}(x) \phi_{1}(x) \\
\varphi_{2}(x) \\
\phi_{2}(x)
\end{array}\right)+\left(\begin{array}{c}
\left(1-\rho_{1}(x)\right) u(x) \\
\left(1-\rho_{1}(x)\right) v(x) \\
0 \\
\left(1-\rho_{1}(x)\right) \varphi_{1}(x) \\
\left(1-\rho_{1}(x)\right) \phi_{1}(x) \\
0 \\
0
\end{array}\right) \triangleq U_{\rho_{1}}(x)+U_{1-\rho_{1}}(x)
$$

and for a matrix $M$, denote $M_{\rho}$ by the same matrix but with its first, second, fourth, and fifth rows multiplied by $\rho(x)$. Hence, from (3.18), we have

$$
U_{\rho_{2}}(x)=R_{\rho_{2}}(x)-A_{\rho_{2}} U_{\rho_{1}}(x)-A_{\rho_{2}} U_{1-\rho_{1}}(x)
$$


The mapping operator $U(x) \rightarrow A_{\rho_{2}} U_{\rho_{1}}(x)$ is bounded from the weighted product space $Y$ into the weighted product space $X$ as its kernel vanishes in a neighborhood of the diagonal element. Moreover, by using Theorems 2.30 and 2.31 in [19], we can see that

$$
\left\|A_{\rho_{2}} U_{1-\rho_{1}}(x)\right\|_{0, \alpha} \leq C\left\|A U_{1-\rho_{1}}(x)\right\|_{0, \alpha} \leq C\left\|U_{1-\rho_{1}}(x)\right\|_{\infty} \leq C\|U(x)\|_{0}
$$

where the norms $\|U(x)\|_{0, \alpha}$ and $\|U(x)\|_{\infty}$ are defined as follows: the first, second, fourth, and fifth components of $U(x)$ are defined by the corresponding norms but its third, sixth, and seventh components are equipped with $C^{1, \alpha}\left(\overline{\Omega_{3}}\right), C^{1, \alpha}\left(S_{2}\right)$ and $C^{0, \alpha}\left(S_{2}\right)$ norms, respectively. From (3.21) and (3.24), we can see that

$$
\begin{aligned}
\|U(x)\|_{0, \alpha} \triangleq & \|u(x)\|_{0, \alpha, \overline{\Omega_{R}} \backslash B_{12}}+\|v(x)\|_{1, \alpha, \overline{\Omega_{2}} \backslash B_{12}}+\|w(x)\|_{0, \alpha, \overline{\Omega_{3}}} \\
& +\left\|\varphi_{1}(x)\right\|_{0, \alpha, S_{1} \backslash B_{12}}+\left\|\phi_{1}(x)\right\|_{0, \alpha, S_{1} \backslash B_{12}}+\left\|\varphi_{2}(x)\right\|_{1, \alpha, S_{2}}+\left\|\phi_{2}(x)\right\|_{0, \alpha, S_{2}} \\
\leq & C\left\|U_{\rho_{2}}(x)\right\|_{0, \alpha} \\
\leq & C\left(\left\|R_{\rho_{2}}(x)\right\|_{0, \alpha}+\|U(x)\|_{0}\right) \\
\leq & C\left(\left\|f_{1}(x)\right\|_{0, S_{1}}+\left\|g_{1}(x)\right\|_{0, S_{1}}+\left\|f_{1}(x)\right\|_{0, \alpha, S_{1} \backslash B_{1}}+\left\|g_{1}(x)\right\|_{0, \alpha, S_{1} \backslash B_{1}}\right. \\
& \left.+\left\|p_{1}(x)\right\|_{1, \alpha, S_{2}}+\left\|q_{1}(x)\right\|_{0, \alpha, S_{2}}+\left\|\Phi_{2}\left(x, z_{1}\right)\right\|_{L^{2}\left(\Omega_{2}\right)}\right) .
\end{aligned}
$$

Then we estimate $\left\|\varphi_{1}(x)\right\|_{1, \alpha, S_{1} \backslash B_{12}}$. Multiplying (3.13) by $\rho_{2}(x)$, using (3.24), and noting the fact that the integral operators mapping $C^{0, \alpha}$-functions into $C^{1, \alpha}$-functions are bounded and the fact that $\varphi_{1}(x)=\rho_{1}(x) \varphi_{1}(x)+\left[1-\rho_{1}(x)\right] \varphi_{1}(x)$ and $\phi_{1}(x)=\rho_{1}(x) \phi_{1}(x)+[1-$ $\left.\rho_{1}(x)\right] \phi_{1}(x)$, we can see that

$$
\begin{aligned}
\left\|\varphi_{1}(x)\right\|_{1, \alpha, S_{1} \backslash B_{12}} \leq & \left\|\rho_{2}(x) \varphi_{1}(x)\right\|_{1, \alpha, S_{1}} \\
\leq & C\left(\left\|\rho_{2}(x)\left[\left(\lambda_{1} K_{1,1}-K_{1,2}\right) \varphi_{1}\right](x)\right\|_{1, \alpha}+\left\|\rho_{2}(x)\left[\left(S_{1,1}-S_{1,2}\right) \phi_{1}\right](x)\right\|_{1, \alpha}\right. \\
& +\left\|\rho_{2}(x) \lambda_{2}\left(K_{2,2} \varphi_{2}\right)(x)\right\|_{1, \alpha}+\left\|\rho_{2}(x)\left(S_{2,2} \phi_{2}\right)(x)\right\|_{1, \alpha} \\
& \left.+\left\|\rho_{2}(x)(V v)(x)\right\|_{1, \alpha}+\left\|\rho_{2}(x) f_{1}(x)\right\|_{1, \alpha}\right) \\
\leq & C\left(\|U(x)\|_{0}+\left\|\left[1-\rho_{1}(x)\right] U(x)\right\|_{0, \alpha}+\left\|\rho_{2}(x) f_{1}(x)\right\|_{1, \alpha}\right) \\
\leq & C\left(\|U(x)\|_{0}+\|U(x)\|_{0, \alpha}+\left\|f_{1}(x)\right\|_{1, \alpha, S_{1} \backslash B_{1}}\right) .
\end{aligned}
$$

From (3.21) and (3.26)-(3.27), we can establish the following estimate in the spaces of Hölder continuous functions for $\left(u(x), v(x), w(x), \varphi_{1}(x), \phi_{1}(x), \varphi_{2}(x), \phi_{2}(x)\right)$ :

$$
\begin{aligned}
\|U(x)\|_{1, \alpha} \triangleq & \|u(x)\|_{0, \alpha, \overline{\Omega_{R}} \backslash B_{12}}+\|v(x)\|_{1, \alpha, \overline{\Omega_{2}} \backslash B_{12}}+\|w(x)\|_{0, \alpha, \overline{\Omega_{3}}} \\
& +\left\|\varphi_{1}(x)\right\|_{1, \alpha, S_{1} \backslash B_{12}}+\left\|\phi_{1}(x)\right\|_{0, \alpha, S_{1} \backslash B_{12}}+\left\|\varphi_{2}(x)\right\|_{1, \alpha, S_{2}}+\left\|\phi_{2}(x)\right\|_{0, \alpha, S_{2}} \\
\leq & C\left(\left\|f_{1}(x)\right\|_{0, S_{1}}+\left\|g_{1}(x)\right\|_{0, S_{1}}+\left\|f_{1}(x)\right\|_{1, \alpha, S_{1} \backslash B_{1}}+\left\|g_{1}(x)\right\|_{0, \alpha, S_{1} \backslash B_{1}}\right. \\
& \left.+\left\|p_{1}(x)\right\|_{1, \alpha, S_{2}}+\left\|q_{1}(x)\right\|_{0, \alpha, S_{2}}+\left\|\Phi_{2}\left(x, z_{1}\right)\right\|_{L^{2}\left(\Omega_{2}\right)}\right) .
\end{aligned}
$$


Next, we estimate $\left\|\frac{\partial v(x)}{\partial v}\right\|_{0, \alpha, S_{1} \backslash B_{2}}$. From (3.11) and the jump relation, we can obtain, on $S_{1}$,

$$
\begin{aligned}
\frac{\partial v(x)}{\partial v}= & \frac{1}{2} \phi_{1}(x)+\left(T_{1,2} \varphi_{1}\right)(x)+\left(K_{1,2}^{\prime} \phi_{1}\right)(x)+\lambda_{2}\left(T_{2,2} \varphi_{2}\right)(x) \\
& +\left(K_{2,2}^{\prime} \phi_{2}\right)(x)+\left(V^{\prime} v\right)(x) .
\end{aligned}
$$

From (3.29) and by using the fact that $\varphi_{1}(x)=\rho_{1}(x) \varphi_{1}(x)+\left[1-\rho_{1}(x)\right] \varphi_{1}(x)$ and $\phi_{1}(x)=$ $\rho_{1}(x) \phi_{1}(x)+\left[1-\rho_{1}(x)\right] \phi_{1}(x)$ again, we can see that

$$
\begin{aligned}
\left\|\frac{\partial v(x)}{\partial v}\right\|_{0, \alpha, S_{1} \backslash B_{2}} \leq & \left\|\left[1-\rho_{1}(x)\right] \frac{\partial v(x)}{\partial v}\right\|_{0, \alpha, S_{1}} \\
\leq & C\left(\|U(x)\|_{0}+\left\|\left[1-\rho_{1}(x)\right] \varphi_{1}(x)\right\|_{1, \alpha, S_{1}}+\left\|\left[1-\rho_{1}(x)\right] \phi_{1}(x)\right\|_{0, \alpha, S_{1}}\right. \\
& \left.+\left\|\varphi_{2}(x)\right\|_{1, \alpha, S_{2}}+\left\|\phi_{2}(x)\right\|_{0, \alpha, S_{2}}\right) \\
\leq & C\left(\|U(x)\|_{0}+\|U(x)\|_{1, \alpha}\right) .
\end{aligned}
$$

From the estimate (3.30) with (3.21) and (3.28), we can see that

$$
\begin{aligned}
\left\|\frac{\partial v(x)}{\partial v}\right\|_{0, \alpha, S_{1} \backslash B_{2}} \leq & C\left(\left\|f_{1}(x)\right\|_{0, S_{1}}+\left\|g_{1}(x)\right\|_{0, S_{1}}+\left\|f_{1}(x)\right\|_{1, \alpha, S_{1} \backslash B_{1}}+\left\|g_{1}(x)\right\|_{0, \alpha, S_{1} \backslash B_{1}}\right. \\
& \left.+\left\|p_{1}(x)\right\|_{1, \alpha, S_{2}}+\left\|q_{1}(x)\right\|_{0, \alpha, S_{2}}+\left\|\Phi_{2}\left(x, z_{1}\right)\right\|_{L^{2}\left(\Omega_{2}\right)}\right) .
\end{aligned}
$$

It finishes the proof of the lemma.

Similarly, let $B_{3}, B_{4}$ be two small balls with center $x^{(2)}$ and radii $r_{3}, r_{4}$, respectively, satisfying that $x^{(2)} \in S_{2}, r_{3}<r_{4}, B_{4} \cap \overline{\Omega_{1}}=\emptyset$. Now we consider the scattering problem of the incident point source $\Phi_{3}\left(x, z_{3}\right)$ with $z_{3} \in B_{3} \cap \Omega_{3}$. To prove the unique determination of the boundary interface $S_{2}$ in the inverse scattering problem in the next section, we need to study the behavior of the solution $w$ on some part of the boundary interface $S_{2}$.

Lemma 5 Assume that $z_{3} \in B_{3} \cap \Omega_{3}$ and $u(x) \in C^{2}\left(\Omega_{1}\right) \cap C^{1, \alpha}\left(\overline{\Omega_{1}}\right), v(x) \in C^{2}\left(\Omega_{2}\right) \cap C^{1, \alpha}\left(\overline{\Omega_{2}}\right)$ and $w(x) \in C^{2}\left(\Omega_{3}\right) \cap C^{1, \alpha}\left(\overline{\Omega_{3}}\right)$ is a solution of the boundary value problem $(2.1)-(2.6)$ with $f_{2}(x), g_{2}(x), p_{2}(x), q_{2}(x)$ given below, then there exists a constant $C>0$ such that

$$
\begin{aligned}
\| w(x) & \left\|_{\infty, S_{2} \backslash B_{4}}+\right\| \frac{\partial w(x)}{\partial v} \|_{\infty, S_{2} \backslash B_{4}} \\
\leq & C\left(\left\|f_{2}(x)\right\|_{1, \alpha, S_{1}}+\left\|g_{2}(x)\right\|_{0, \alpha, S_{1}}+\left\|p_{2}(x)\right\|_{0, S_{2}}+\left\|q_{2}(x)\right\|_{0, S_{2}}\right. \\
& \left.+\left\|p_{2}(x)\right\|_{1, \alpha, S_{2} \backslash B_{3}}+\left\|q_{2}(x)\right\|_{0, \alpha, S_{2} \backslash B_{3}}\right),
\end{aligned}
$$

where $f_{2}(x)=\frac{1}{\lambda_{1} \lambda_{2}} \Phi_{3}\left(x, z_{3}\right), g_{2}(x)=\frac{\partial \Phi_{3}\left(x, z_{3}\right)}{\partial v}, p_{2}(x)=-\frac{1}{\lambda_{2}} \Phi_{3}\left(x, z_{3}\right), q_{2}(x)=-\frac{\partial \Phi_{3}\left(x, z_{3}\right)}{\partial v}$.

Proof Arguing similarly as in the above lemma, in addition to the weighted product space $X, Y$, we also consider the weighted product space

$$
Z \triangleq C_{0}\left(\overline{\Omega_{R}}\right) \times C_{0}\left(\overline{\Omega_{2}}\right) \times C^{1, \alpha}\left(\overline{\Omega_{3}}\right) \times C^{1, \alpha}\left(S_{1}\right) \times C^{0, \alpha}\left(S_{1}\right) \times C_{0}\left(S_{2}\right) \times C_{0}\left(S_{2}\right) .
$$


From [20] and [13], we know that all entries of the matrix operator $A$ are compact, hence, we can easily see that the matrix operator $A$ is compact in the weighted product space $Z$. By using Theorem 2 in [18], we know that the operator $I+A$ has a trivial null space in the weighted product space $X$. Consequently, by applying the Fredholm alternative to the dual system $\langle X, Z\rangle$ with the $L^{2}$ bilinear form, we can see that the adjoint operator $I+A^{\prime}$ has a trivial null space in the weighted product space $Z$. Then, by applying the Fredholm alternative again to the dual system $\langle Z, Z\rangle$ with the $L^{2}$ bilinear form, we can see that the operator $I+A$ has a trivial null space in the weighted product space $Z$. Hence, by using the Riesz-Fredholm theory, system (3.18) is uniquely solvable in the weighted product space $Z$, and the solution depends continuously on the right-hand side:

$$
\begin{aligned}
\|U(x)\|_{0} \triangleq & \|u(x)\|_{0, \overline{\Omega_{R}}}+\|v(x)\|_{0, \overline{\Omega_{2}}}+\|w(x)\|_{1, \alpha, \overline{\Omega_{3}}} \\
& +\left\|\varphi_{1}(x)\right\|_{1, \alpha, S_{1}}+\left\|\phi_{1}(x)\right\|_{0, \alpha, S_{1}}+\left\|\varphi_{2}(x)\right\|_{0, S_{2}}+\left\|\phi_{2}(x)\right\|_{0, S_{2}} \\
\leq & C\left(\left\|f_{2}(x)\right\|_{1, \alpha, S_{1}}+\left\|g_{2}(x)\right\|_{0, \alpha, S_{1}}+\left\|p_{2}(x)\right\|_{0, S_{2}}+\left\|q_{2}(x)\right\|_{0, S_{2}}\right) .
\end{aligned}
$$

In particular, this implies that

$$
\|w(x)\|_{\infty, S_{2} \backslash B_{4}} \leq C\left(\left\|f_{2}(x)\right\|_{1, \alpha, S_{1}}+\left\|g_{2}(x)\right\|_{0, \alpha, S_{1}}+\left\|p_{2}(x)\right\|_{0, S_{2}}+\left\|q_{2}(x)\right\|_{0, S_{2}}\right) .
$$

Let $B_{34}$ be a ball of radius $r_{34}$ and centered at $x^{(2)}$ with $r_{3}<r_{34}<r_{4}$, and assume that $\rho_{3}(x) \in C^{2}\left(S_{2}\right)$ is a function satisfying $\rho_{3}(x)=0$ for $x \in S_{2} \backslash B_{4}$ and $\rho_{3}(x)=1$ in the neighborhood of $B_{34}, \rho_{4}(x) \in C^{2}\left(S_{2}\right)$ is another function satisfying $\rho_{4}(x)=0$ for $x \in S_{2} \backslash B_{34}$ and $\rho_{4}(x)=1$ in the neighborhood of $B_{3}$.

We rewrite $U(x)$ in the form

$$
\begin{aligned}
U & =\left(\begin{array}{c}
u(x) \\
v(x) \\
w(x) \\
\varphi_{1}(x) \\
\phi_{1}(x) \\
\varphi_{2}(x) \\
\phi_{2}(x)
\end{array}\right)=\left(\begin{array}{c}
u(x) \\
\rho_{3}(x) v(x) \\
\rho_{3}(x) w(x) \\
\varphi_{1}(x) \\
\phi_{1}(x) \\
\rho_{3}(x) \varphi_{2}(x) \\
\rho_{3}(x) \phi_{2}(x)
\end{array}\right)+\left(\begin{array}{c}
0 \\
\left(1-\rho_{3}(x)\right) v(x) \\
\left(1-\rho_{3}(x)\right) w(x) \\
0 \\
0 \\
\left(1-\rho_{3}(x)\right) \varphi_{2}(x) \\
\left(1-\rho_{3}(x)\right) \phi_{2}(x)
\end{array}\right) \\
& \triangleq U_{\rho_{3}}(x)+U_{1-\rho_{3}}(x),
\end{aligned}
$$

and for a matrix $N$, denote $N_{\rho}$ by the same matrix but with its second, third, sixth, and seventh rows multiplied by $\rho(x)$. Hence, from (3.18), we have

$$
U_{\rho_{4}}(x)=R_{\rho_{4}}(x)-A_{\rho_{4}} U_{\rho_{3}}(x)-A_{\rho_{4}} U_{1-\rho_{3}}(x)
$$

The mapping operator $U(x) \rightarrow A_{\rho_{4}} U_{\rho_{3}}(x)$ is bounded from the weighted product space $Z$ into the weighted product space $X$ since its kernel vanishes in a neighborhood of the diagonal element. Moreover, by using theorems 2.30 and 2.31 in [19], we can see that

$$
\left\|A_{\rho_{4}} U_{1-\rho_{3}}(x)\right\|_{0, \alpha} \leq C\left\|A U_{1-\rho_{3}}(x)\right\|_{0, \alpha} \leq C\left\|U_{1-\rho_{3}}(x)\right\|_{\infty} \leq C\|U(x)\|_{0}
$$


where the norms $\|U(x)\|_{0, \alpha}$ and $\|U(x)\|_{\infty}$ are defined as follows: the second, third, sixth, and seventh components of $U(x)$ are defined by the corresponding norms but its first, fourth, and fifth components are equipped with $C^{1, \alpha}\left(\overline{\Omega_{R}}\right), C^{1, \alpha}\left(S_{1}\right)$ and $C^{0, \alpha}\left(S_{1}\right)$ norms, respectively. From (3.34) and (3.37), we can see that

$$
\begin{aligned}
\|U(x)\|_{0, \alpha} \triangleq & \|u(x)\|_{0, \alpha, \overline{\Omega_{R}}}+\|v(x)\|_{1, \alpha, \overline{\Omega_{2}} \backslash B_{34}}+\|w(x)\|_{0, \alpha, \overline{\Omega_{3}} \backslash B_{34}} \\
& +\left\|\varphi_{1}(x)\right\|_{1, \alpha, S_{1}}+\left\|\phi_{1}(x)\right\|_{0, \alpha, S_{1}}+\left\|\varphi_{2}(x)\right\|_{0, \alpha, S_{2} \backslash B_{34}}+\left\|\phi_{2}(x)\right\|_{0, \alpha, S_{2} \backslash B_{3}} \\
\leq & C\left\|U_{\rho_{4}}(x)\right\|_{0, \alpha} \\
\leq & C\left(\left\|R_{\rho_{4}}(x)\right\|_{0, \alpha}+\|U(x)\|_{0}\right) \\
\leq & C\left(\left\|f_{2}(x)\right\|_{1, \alpha, S_{1}}+\left\|g_{2}(x)\right\|_{0, \alpha, S_{1}}+\left\|p_{2}(x)\right\|_{1, \alpha, S_{2} \backslash B_{3}}+\left\|q_{2}(x)\right\|_{0, \alpha, S_{2} \backslash B_{3}}\right. \\
& \left.+\left\|p_{2}(x)\right\|_{0, S_{2}}+\left\|q_{2}(x)\right\|_{0, S_{2}}\right) .
\end{aligned}
$$

Then we estimate $\left\|\varphi_{2}(x)\right\|_{1, \alpha, S_{2} \backslash B_{34}}$. Multiplying (3.15) by $\rho_{4}(x)$, using (3.37), and noting the fact that the integral operators mapping $C^{0, \alpha}$-functions into $C^{1, \alpha}$-functions are bounded and the fact that $\varphi_{2}(x)=\rho_{3}(x) \varphi_{2}(x)+\left[1-\rho_{3}(x)\right] \varphi_{2}(x)$ and $\phi_{2}(x)=\rho_{3}(x) \phi_{2}(x)+[1-$ $\left.\rho_{3}(x)\right] \phi_{2}(x)$, we can see that

$$
\begin{aligned}
\left\|\varphi_{2}(x)\right\|_{1, \alpha, S_{2} \backslash B_{34} \leq} \leq & \left\|\rho_{4}(x) \varphi_{2}(x)\right\|_{1, \alpha, S_{2}} \\
\leq & C\left(\left\|\rho_{4}(x)\left[\left(\lambda_{2} K_{2,2}-K_{2,3}\right) \varphi_{2}\right](x)\right\|_{1, \alpha}+\left\|\rho_{4}(x)\left[\left(S_{2,2}-S_{2,3}\right) \phi_{2}\right](x)\right\|_{1, \alpha}\right. \\
& +\left\|\rho_{4}(x)\left(K_{1,2} \varphi_{1}\right)(x)\right\|_{1, \alpha}+\left\|\rho_{4}(x)\left(S_{1,2} \phi_{1}\right)(x)\right\|_{1, \alpha} \\
& \left.+\left\|\rho_{4}(x)(V v)(x)\right\|_{1, \alpha}+\left\|\rho_{4}(x) p_{2}(x)\right\|_{1, \alpha}\right) \\
\leq & C\left(\|U(x)\|_{0}+\left\|\left[1-\rho_{3}(x)\right] U(x)\right\|_{0, \alpha}+\left\|\rho_{4}(x) p_{2}(x)\right\|_{1, \alpha}\right) \\
\leq & C\left(\|U(x)\|_{0}+\|U(x)\|_{0, \alpha}+\left\|p_{2}(x)\right\|_{1, \alpha, S_{2} \backslash B_{3}}\right)
\end{aligned}
$$

From (3.34) and (3.39)-(3.40), we can establish the following estimate in the spaces of Hölder continuous functions for $\left(u(x), v(x), w(x), \varphi_{1}(x), \phi_{1}(x), \varphi_{2}(x), \phi_{2}(x)\right)$ :

$$
\begin{aligned}
\|U(x)\|_{1, \alpha} \triangleq & \|u(x)\|_{0, \alpha, \overline{\Omega_{R}}}+\|v(x)\|_{1, \alpha, \overline{\Omega_{2}} \backslash B_{34}}+\|w(x)\|_{0, \alpha, \overline{\Omega_{3}} \backslash B_{34}} \\
& +\left\|\varphi_{1}(x)\right\|_{1, \alpha, S_{1}}+\left\|\phi_{1}(x)\right\|_{0, \alpha, S_{1}}+\left\|\varphi_{2}(x)\right\|_{1, \alpha, S_{2} \backslash B_{34}}+\left\|\phi_{2}(x)\right\|_{0, \alpha, S_{2} \backslash B_{34}} \\
\leq & C\left(\left\|f_{2}(x)\right\|_{1, \alpha, S_{1}}+\left\|g_{2}(x)\right\|_{0, \alpha, S_{1}}+\left\|p_{2}(x)\right\|_{1, \alpha, S_{2} \backslash B_{3}}+\left\|q_{2}(x)\right\|_{0, \alpha, S_{2} \backslash B_{3}}\right. \\
& \left.+\left\|p_{2}(x)\right\|_{0, S_{2}}+\left\|q_{2}(x)\right\|_{0, S_{2}}\right) .
\end{aligned}
$$

Next, we estimate $\left\|\frac{\partial w(x)}{\partial v}\right\|_{0, \alpha, S_{2} \backslash B_{4}}$. From (3.12) and the jump relation, we can see that, on $S_{2}$,

$$
\frac{\partial w(x)}{\partial v}=\frac{1}{2} \phi_{2}(x)+\left(T_{2,3} \varphi_{2}\right)(x)+\left(K_{2,3}^{\prime} \phi_{2}\right)(x) .
$$


From (3.42), and by using the fact that $\varphi_{2}(x)=\rho_{3}(x) \varphi_{2}(x)+\left[1-\rho_{3}(x)\right] \varphi_{2}(x)$ and $\phi_{2}(x)=$ $\rho_{3}(x) \phi_{2}(x)+\left[1-\rho_{3}(x)\right] \phi_{2}(x)$ again, we can see that

$$
\begin{aligned}
\left\|\frac{\partial w(x)}{\partial v}\right\|_{0, \alpha, S_{2} \backslash B_{4}} & \leq\left\|\left[1-\rho_{3}(x)\right] \frac{\partial w(x)}{\partial v}\right\|_{0, \alpha, S_{2}} \\
& \leq C\left(\|U(x)\|_{0}+\left\|\left[1-\rho_{3}(x)\right] \varphi_{2}(x)\right\|_{1, \alpha, S_{2}}+\left\|\left[1-\rho_{3}(x)\right] \phi_{2}(x)\right\|_{0, \alpha, S_{2}}\right) \\
& \leq C\left(\|U(x)\|_{0}+\|U(x)\|_{1, \alpha}\right) .
\end{aligned}
$$

From the estimate (3.43) with (3.34) and (3.41), we can see that

$$
\begin{aligned}
\left\|\frac{\partial w(x)}{\partial v}\right\|_{0, \alpha, S_{2} \backslash B_{4}} \leq & C\left(\left\|f_{2}(x)\right\|_{1, \alpha, S_{1}}+\left\|g_{2}(x)\right\|_{0, \alpha, S_{1}}+\left\|p_{2}(x)\right\|_{1, \alpha, S_{2} \backslash B_{3}}+\left\|q_{2}(x)\right\|_{0, \alpha, S_{2} \backslash B_{3}}\right. \\
& \left.+\left\|p_{2}(x)\right\|_{0, S_{2}}+\left\|q_{2}(x)\right\|_{0, S_{2}}\right) .
\end{aligned}
$$

It finishes the proof of the lemma.

\section{Conclusions}

In this section, we will prove that both the penetrable interfaces $S_{i}(i=1,2)$ and the refractive index $n(x)$ of the inhomogeneous penetrable obstacle $\Omega_{2}$ can be uniquely determined from knowledge of the far-field pattern $u^{\infty}(\widehat{x}, d)(\widehat{x}, d \in \mathbb{S})$ for incident plane waves $u^{i}(x)=e^{i k_{1} x \cdot d}$.

\subsection{Unique determination of the penetrable interfaces $S_{i}(i=1,2)$}

Following the transmission boundary value problems in a homogeneous medium [20], the transmission boundary value problems in an inhomogeneous medium [13] and the inhomogeneous impenetrable obstacle scattering in a stratified medium [9], we prove in this subsection that the penetrable interfaces $S_{i}(i=1,2)$ can be uniquely determined by the far-field pattern $u^{\infty}(\widehat{x}, d)(\widehat{x}, d \in \mathbb{S})$ for incident plane waves $u^{i}(x)=e^{i k_{1} x \cdot d}$. To achieve this, we first give the following two lemmas.

Lemma 6 Assume that $h_{1}(x) \in L^{2}\left(\Omega_{2}\right), g_{3}(x) \in C\left(S_{1}\right), p_{3}(x) \in C\left(S_{2}\right), q_{3}(x) \in C\left(S_{2}\right), \eta_{1}(x) \in$ $C\left(S_{1}\right)$ with $\eta_{1}(x) \neq 0$ and $\eta_{1}(x) \leq 0$ on $S_{1}$, then the following boundary value problem has a unique solution $v(x) \in C^{2}\left(\Omega_{2}\right) \cap C\left(\overline{\Omega_{2}}\right)$ and $w(x) \in C^{2}\left(\Omega_{3}\right) \cap C\left(\overline{\Omega_{3}}\right)$ :

$$
\begin{aligned}
& \Delta v(x)+k_{2}^{2} n(x) v(x)=h_{1}(x), \quad \text { in } \Omega_{2}, \\
& \Delta w(x)+k_{3}^{2} w(x)=0, \quad \text { in } \Omega_{3}, \\
& \frac{\partial v(x)}{\partial v}+i \eta_{1}(x) v(x)=g_{3}(x), \quad \text { on } S_{1}, \\
& v(x)-w(x)=p_{3}(x), \quad \frac{\partial v(x)}{\partial v}-\lambda_{2} \frac{\partial w(x)}{\partial v}=q_{3}(x), \quad \text { on } S_{2} .
\end{aligned}
$$

Moreover, there exists a constant $C>0$ such that

$$
\|v(x)\|_{\infty, \overline{\Omega_{2}}} \leq C\left(\left\|h_{1}(x)\right\|_{L^{2}\left(\Omega_{2}\right)}+\left\|g_{3}(x)\right\|_{\infty, S_{1}}+\left\|p_{3}(x)\right\|_{\infty, S_{2}}+\left\|q_{3}(x)\right\|_{\infty, S_{2}}\right) .
$$


Proof First, we prove the uniqueness of solutions, that is $v(x)=0$ in $\Omega_{2}, w(x)=0$ in $\Omega_{3}$ if $h_{1}(x)=0$ in $\Omega_{2}, g_{3}(x)=0$ on $S_{1}, p_{3}(x)=q_{3}(x)=0$ on $S_{2}$. From equations (4.1)-(4.2), boundary conditions (4.3)-(4.4), noting the assumption that $k_{2}^{2}$ is not a Neumann eigenvalue of $\Delta v(x)+k_{2}^{2} n(x) v(x)=0$ in $\Omega_{2}$, and using Green's first theorem over $\Omega_{2}$ and $\Omega_{3}$, we can see that

$$
\begin{aligned}
0= & \int_{\Omega_{2}}\left\{\left[\Delta v(x)+k_{2}^{2} n(x) v(x)\right] \bar{v}(x)\right\} d x \\
= & \int_{\Omega_{2}}\left[-|\nabla v(x)|^{2}+k_{2}^{2} n(x)|v(x)|^{2}\right] d x+\int_{S_{1}} \bar{v}(x) \frac{\partial v(x)}{\partial v} d s-\int_{S_{2}} \bar{v}(x) \frac{\partial v(x)}{\partial v} d s \\
= & \int_{\Omega_{2}}\left[-|\nabla v(x)|^{2}+k_{2}^{2} n(x)|v(x)|^{2}\right] d x \\
& -i \int_{S_{1}} \eta_{1}(x)|v(x)|^{2} d s-\lambda_{2} \int_{S_{2}} \bar{w}(x) \frac{\partial w(x)}{\partial v} d s \\
= & \int_{\Omega_{2}}\left[-|\nabla v(x)|^{2}+k_{2}^{2} n(x)|v(x)|^{2}\right] d x-i \int_{S_{1}} \eta_{1}(x)|v(x)|^{2} d s \\
& -\lambda_{2} \int_{\Omega_{3}}\left[|\nabla w(x)|^{2}+\Delta w(x) \cdot \bar{w}(x)\right] d x \\
= & \int_{\Omega_{2}}\left[-|\nabla v(x)|^{2}+k_{2}^{2} n(x)|v(x)|^{2}\right] d x-i \int_{S_{1}} \eta_{1}(x)|v(x)|^{2} d s \\
& -\lambda_{2} \int_{\Omega_{3}}\left[|\nabla w(x)|^{2}-k_{3}^{2}|w(x)|^{2}\right] d x .
\end{aligned}
$$

By taking the imaginary part of the above equation (4.6), we can see that $v(x)=0$ on some part $\Gamma_{1}$ of $S_{1}$ since $\eta_{1}(x) \neq 0, \eta_{1}(x) \leq 0$ on $S_{1}, \lambda_{2}>0$ is a given number and $\Im[n(x)] \geq 0$. From the boundary condition (4.3), we can see that $\frac{\partial v(x)}{\partial v}=-i \eta_{1}(x) v(x)=0$ on some part $\Gamma_{1}$ of $S_{1}$. Therefore, by using Holmgren's uniqueness theorem [21], we can see that $v(x)=0$ in $\Omega_{2}$. By using the transmission boundary conditions (4.4), we have $w(x)=0$ in $\Omega_{3}$.

Then we prove the existence of solutions. To achieve this, we introduce the volume potential

$$
\left(V^{\star} h_{1}\right)(x) \triangleq \int_{\Omega_{2}} \Phi_{2}(x, y) h_{1}(y) d y, \quad x \in \Omega_{2}
$$

from Theorem 8.2 [19], we know this is a bounded operator $V^{\star}: L^{2}\left(\Omega_{2}\right) \rightarrow H^{2}\left(\Omega_{2}\right)$. Now we seek a solution in the form

$$
\begin{aligned}
v(x)= & -\left(V^{\star} h_{1}\right)(x)+\left[\widetilde{S}_{1,2}\left(\varphi_{3}+\varphi_{4}\right)\right](x)+\lambda_{2}\left[\widetilde{K}_{2,2}\left(\phi_{3}+\phi_{4}\right)\right](x) \\
& +\left[\widetilde{S}_{2,2}\left(\psi_{1}+\psi_{2}\right)\right](x)+\left(V^{\star} v\right)(x), \quad \text { in } \Omega_{2}, \\
w(x)= & {\left[\widetilde{K}_{2,3}\left(\phi_{3}+\phi_{4}\right)\right](x)+\left[\widetilde{S}_{2,3}\left(\psi_{1}+\psi_{2}\right)\right](x), \quad \text { in } \Omega_{3}, }
\end{aligned}
$$

with six densities $\varphi_{3}(x) \in C\left(S_{1}\right), \varphi_{4}(x) \in H^{\frac{1}{2}}\left(S_{1}\right), \phi_{3}(x) \in C\left(S_{2}\right), \phi_{4}(x) \in H^{\frac{1}{2}}\left(S_{2}\right), \psi_{1}(x) \in$ $C\left(S_{2}\right), \psi_{2}(x) \in H^{\frac{1}{2}}\left(S_{2}\right)$. From the jump conditions, we can see that the potentials $v(x), w(x)$ given by (4.8) and (4.9) solve the boundary value problem (4.1)-(4.4) if the six mentioned 
densities satisfy the following system of integral equations:

$$
\begin{aligned}
& \frac{1}{2} \varphi_{3}(x)+\left[\left(K_{1,2}^{\prime}+i \eta_{1} S_{1,2}\right) \varphi_{3}\right](x)+\lambda_{2}\left[\left(T_{2,2}+i \eta_{1} K_{2,2}\right)\left(\phi_{3}+\phi_{4}\right)\right](x) \\
& \quad+\left[\left(K_{2,2}^{\prime}+i \eta_{1} S_{2,2}\right)\left(\psi_{1}+\psi_{2}\right)\right](x)=g_{3}(x), \quad \text { on } S_{1} \\
& \frac{1}{2} \varphi_{4}(x)+\left[\left(K_{1,2}^{\prime}+i \eta_{1} S_{1,2}\right) \varphi_{4}\right](x)=\frac{\partial\left(V^{\star} h_{1}-V^{\star} v\right)(x)}{\partial v}+i \eta_{1}(x)\left(V^{\star} h_{1}-V^{\star} v\right)(x) \\
& \quad \text { on } S_{1} \\
& \frac{\lambda_{2}+1}{2} \phi_{3}(x)+\left[S_{1,2}\left(\varphi_{3}+\varphi_{4}\right)\right](x)+\left[\left(\lambda_{2} K_{2,2}-K_{2,3}\right) \phi_{3}\right](x)+\left[\left(S_{2,2}-S_{2,3}\right) \psi_{1}\right](x)=p_{3}(x) \\
& \quad \text { on } S_{2}, \\
& \frac{\lambda_{2}+1}{2} \phi_{4}(x)+\left[\left(\lambda_{2} K_{2,2}-K_{2,3}\right) \phi_{4}\right](x)+\left[\left(S_{2,2}-S_{2,3}\right) \psi_{2}\right](x)=\left(V^{\star} h_{1}-V^{\star} v\right)(x) \\
& \quad \text { on } S_{2}, \\
& -\frac{\lambda_{2}+1}{2} \psi_{1}(x)+\left[K_{1,2}^{\prime}\left(\varphi_{3}+\varphi_{4}\right)\right](x)+\lambda_{2}\left[\left(T_{2,2}-T_{2,3}\right) \phi_{3}\right](x) \\
& \quad+\left[\left(K_{2,2}^{\prime}-\lambda_{2} K_{2,3}^{\prime}\right) \psi_{1}\right](x)=q_{3}(x), \quad \text { on } S_{2} \\
& -\frac{\lambda_{2}+1}{2} \psi_{2}(x)+\left[\left(K_{2,2}^{\prime}-\lambda_{2} K_{2,3}^{\prime}\right) \psi_{2}\right](x)+\lambda_{2}\left[\left(T_{2,2}-T_{2,3}\right) \phi_{4}\right](x) \\
& \quad=\frac{\partial\left(V^{\star} h_{1}--V^{\star} v\right)(x)}{\partial v}, \quad \text { on } S_{2} \text {. }
\end{aligned}
$$

Next, we look for a solution $\left(v(x), w(x), \varphi_{3}(x), \varphi_{4}(x), \phi_{3}(x), \phi_{4}(x), \psi_{1}(x), \psi_{2}(x)\right) \in W$ to the above system of eight integral equations, where $W$ is a weighted product space defined as follows:

$$
W \triangleq C\left(\overline{\Omega_{2}}\right) \times C\left(\overline{\Omega_{3}}\right) \times C\left(S_{1}\right) \times H^{\frac{1}{2}}\left(S_{1}\right) \times C\left(S_{2}\right) \times H^{\frac{1}{2}}\left(S_{2}\right) \times C\left(S_{2}\right) \times H^{\frac{1}{2}}\left(S_{2}\right)
$$

By using the uniqueness of solutions to the problem and standard arguments, we can easily see that this system has at most one solution in the weighted product space $W$. Thus, by using the Riesz-Fredholm theory, we can easily obtain the existence of solutions to the boundary value problem with the estimate:

$$
\begin{aligned}
\|v(x)\|_{\infty, \overline{\Omega_{2}}} \leq & C\left(\left\|\left(V^{\star} h_{1}\right)(x)\right\|_{\infty, \overline{\Omega_{2}}}+\left\|\frac{\partial\left(V^{\star} h_{1}\right)(x)}{\partial v}+i \eta_{1}(x)\left(V^{\star} h_{1}\right)(x)\right\|_{H^{\frac{1}{2}}\left(S_{1} \cup S_{2}\right)}\right. \\
& \left.+\left\|g_{3}(x)\right\|_{\infty, S_{1}}+\left\|p_{3}(x)\right\|_{\infty, S_{2}}+\left\|q_{3}(x)\right\|_{\infty, S_{2}}\right) \\
\leq & C\left(\left\|h_{1}(x)\right\|_{L^{2}\left(\Omega_{2}\right)}+\left\|g_{3}(x)\right\|_{\infty, S_{1}}+\left\|p_{3}(x)\right\|_{\infty, S_{2}}+\left\|q_{3}(x)\right\|_{\infty, S_{2}}\right),
\end{aligned}
$$

for some constant $C>0$. It finishes the proof of the lemma.

Lemma 7 Assume that $h_{2}(x) \in L^{2}\left(\Omega_{3}\right), q_{4}(x) \in C\left(S_{2}\right), \eta_{2}(x) \in C\left(S_{2}\right)$ with $\eta_{2}(x) \neq 0$ and $\eta_{2}(x) \leq 0$ on $S_{2}$, then the following boundary value problem has a unique solution $w(x) \in$ 
$C^{2}\left(\Omega_{3}\right) \cap C\left(\overline{\Omega_{3}}\right):$

$$
\begin{aligned}
& \Delta w(x)+k_{3}^{2} w(x)=h_{2}(x), \quad \text { in } \Omega_{3}, \\
& \frac{\partial w(x)}{\partial v}+i \eta_{2}(x) w(x)=q_{4}(x), \quad \text { on } S_{2} .
\end{aligned}
$$

Furthermore, there exists a constant $C>0$ such that

$$
\|w(x)\|_{\infty, \overline{\Omega_{3}}} \leq C\left(\left\|h_{2}(x)\right\|_{L^{2}\left(\Omega_{3}\right)}+\left\|q_{4}(x)\right\|_{\infty, S_{2}}\right) .
$$

Proof The proof is analogous to part of the proof of Lemma 4.4 in [13] and, hence, is omitted.

So we can obtain our first result as follows.

Theorem 1 Assume that $\lambda_{1} \neq 1, \lambda_{2} \neq 1$, let $S_{1}, \widetilde{S}_{1}$ be two penetrable interfaces and let $\Omega_{2}, \widetilde{\Omega}_{2}$ be two penetrable obstacles for the corresponding scattering problem. If the far-field patterns $u^{\infty}(\widehat{x}, d)(\widehat{x}, d \in \mathbb{S})$ of the scattered fields for the same incident plane wave $u^{i}(x)=e^{i k_{1} x \cdot d}$ coincide at a fixed frequency for every incident direction $d \in \mathbb{S}$ and the observation direction $\widehat{x} \in \mathbb{S}$, then $S_{1}=\widetilde{S}_{1}, S_{2}=\widetilde{S}_{2}$.

Proof Using Lemmas 3, 4, 5, 6, and 7, and arguing analogously to part of the proof of Theorem 3.2 in [9], we can easily prove the uniqueness result.

\subsection{Unique determination of the refractive index $n(x)$ of the inhomogeneous penetrable obstacle $\boldsymbol{\Omega}_{2}$}

In this subsection, we will prove a uniqueness theorem for reconstructing the refractive index $n(x)$ of the inhomogeneous penetrable obstacle $\Omega_{2}$ from the far-field pattern $u^{\infty}(\widehat{x}, d)$ $(\widehat{x}, d \in \mathbb{S})$ for incident plane waves $u^{i}(x)=e^{i k_{1} x \cdot d}$. To do this, we need the following two lemmas: one is the completeness result, the other is the orthogonality relation.

Lemma 8 Assume that $k_{2}^{2}$ is not a Neumann eigenvalue of $\Delta v(x)+k_{2}^{2} n(x) v(x)=0$ in $\Omega_{2}$, then the set $\left\{\frac{\partial v(x, d)}{\partial v} \mid d \in \mathbb{S}\right\}$ of normal derivatives of the fields $v(x, d)$ are complete in $L^{2}\left(S_{1}\right)$, where the fields $v(x, d)$ correspond to incident plane waves $u^{i}(x)=e^{i k_{1} x \cdot d}, d \in \mathbb{S}$ is the incident direction.

Proof Denote $\Omega$ by the complement of $\Omega_{1}$, that is, $\Omega \triangleq \mathbb{R}^{3} \backslash \overline{\Omega_{1}}$. Choose a large ball $B_{R}$ centered at the origin such that $\bar{\Omega} \subset B_{R}$ and such that $k_{1}^{2}$ is not a Dirichlet eigenvalue of $\Delta u(x)+k_{1}^{2} u(x)=0$ in $B_{R}$, then, from Theorem 5.5 in [6], we know that the restriction to $\partial B_{R}$ of the set of plane waves $u^{i}(x)=e^{i k_{1} x \cdot d}$ are complete in $L^{2}\left(\partial B_{R}\right)$. Therefore, we just need to prove that the operator $I I: L^{2}\left(\partial B_{R}\right) \rightarrow L^{2}\left(S_{1}\right)$ has a dense range $I I \varphi(x)=\frac{\partial v(x)}{\partial v}$, where $v(x)$ solves the boundary value problem (1.1)-(1.6) and $\varphi(x)$ is the boundary data of the following interior Dirichlet problem:

$$
\Delta u^{i}(x)+k_{1}^{2} u^{i}(x)=0 \quad \text { in } B_{R}, \quad u^{i}(x)=\varphi(x) \quad \text { on } \partial B_{R} .
$$

By using Green's formulas, we can see that the $L^{2}$-adjoint $I I^{\prime}$ of $I I$ is given by

$$
\left.I I^{\prime} \phi(x) \triangleq \overline{\left\{\frac{\partial \widehat{u}(x)}{\partial v}-\frac{\partial \widetilde{u}(x)}{\partial v}\right\}}\right|_{\partial B_{R}}, \quad \phi(x) \in L^{2}\left(S_{1}\right)
$$


where $(\widehat{u}(x), \widehat{v}(x))$ solves

$$
\begin{aligned}
& \Delta \widehat{u}(x)+k_{1}^{2} \widehat{v}(x)=0, \quad \text { in } \Omega_{1}, \\
& \Delta \widehat{v}(x)+k_{2}^{2} n(x) \widehat{v}(x)=0, \quad \text { in } \Omega_{2}, \\
& \widehat{u}(x)-\widehat{v}(x)=\phi(x), \quad \frac{\partial \widehat{u}(x)}{\partial v}-\lambda_{1} \frac{\partial \widehat{v}(x)}{\partial v}=0, \quad \text { on } S_{1}, \\
& \lim _{r \rightarrow \infty} r\left(\frac{\partial \widehat{u}(x)}{\partial r}-i k_{1} \widehat{u}(x)\right)=0,
\end{aligned}
$$

and $\widetilde{u}(x)$ is a solution of the following interior Dirichlet problem:

$$
\Delta \widetilde{u}(x)+k_{1}^{2} \widetilde{u}(x)=0 \quad \text { in } B_{R}, \quad \widetilde{u}(x)=\widehat{u}(x) \quad \text { on } \partial B_{R} .
$$

We only have to prove that $I I^{\prime}$ is injective. Let $I I^{\prime} \phi(x)=0$, then we know that $\frac{\partial \widehat{u}(x)}{\partial v}=\frac{\partial \widetilde{u}(x)}{\partial v}$ and $\widehat{u}(x)=\widetilde{u}(x)$ on $\partial B_{R}$. Define

$$
\widetilde{v}(x)= \begin{cases}\widehat{u}(x), & \text { in } \mathbb{R}^{3} \backslash \overline{B_{R}} \\ \widetilde{u}(x), & \text { in } B_{R} .\end{cases}
$$

Then, from [8], we know that $\widetilde{v}(x)$ is an entire solution to the Helmholtz equation $\Delta \widetilde{v}(x)+$ $k_{1}^{2} \widetilde{v}(x)=0$ in $\mathbb{R}^{3}$ satisfying the radiation condition (4.26), so it must vanish identically in $\mathbb{R}^{3}$. Therefore, $\widehat{u}(x)=0$ in $\mathbb{R}^{3} \backslash \overline{B_{R}}$. By using the unique continuation principle, we can see that $\widehat{u}(x)=0$ in $\Omega_{1}$. From the transmission conditions (4.25), Holmgren's uniqueness theorem and by noting the assumption that $k_{2}^{2}$ is not a Neumann eigenvalue of $\Delta v(x)+$ $k_{2}^{2} n(x) v(x)=0$ in $\Omega_{2}$, we can see that $\widehat{v}(x)=0$ in $\Omega_{2}$ and, hence, $\frac{\partial \widehat{v}(x)}{\partial v}=\widehat{v}(x)=0$ on $S_{1}$. It finishes the proof of the lemma.

Lemma 9 Assume that the far-field patterns $u^{\infty}(\widehat{x}, d)(\widehat{x}, d \in \mathbb{S})$ for the refractive indices $n(x)$ and $\widetilde{n}(x)$ coincide, then, for any solution $v(x) \in C^{2}\left(\overline{\Omega_{2}}\right) \cap C\left(\Omega_{2}\right)$ of $\Delta v(x)+k_{2}^{2} n(x) v(x)=$ 0 in $\Omega_{2}$ and any solution $\widetilde{v}(x) \in C^{2}\left(\overline{\Omega_{2}}\right) \cap C\left(\Omega_{2}\right)$ of $\Delta \widetilde{v}(x)+k_{2}^{2} \widetilde{n}(x) \widetilde{v}(x)=0$ in $\Omega_{2}$, we have the following orthogonality relation:

$$
\int_{\Omega_{2}}[n(x)-\widetilde{n}(x)] v(x) \widetilde{v}(x) d x=0 .
$$

Proof First, we prove (4.29) for the special case $v(x)=v(x, d), d \in \mathbb{S}$. By using Rellich's lemma and Holmgren's uniqueness theorem, we can see that $v(x, d)=\widetilde{v}(x, d)$ and $\frac{\partial v(x, d)}{\partial v}=$ $\frac{\widetilde{v}(x, d)}{\partial v}$ on $S_{1}$. By using Green's first theorem and the equations satisfied by $v(x, d)$ and $\widetilde{v}(x, d)$, we can see that

$$
\begin{aligned}
\int_{\Omega_{2}} & {[n(x)-\widetilde{n}(x)] v(x, d) \widetilde{v}(x) d x } \\
= & -\frac{1}{k_{2}^{2}} \int_{\Omega_{2}}\left[\Delta v(x, d)+k_{2}^{2} \widetilde{n}(x) v(x, d)\right] \widetilde{v}(x) d x \\
= & -\frac{1}{k_{2}^{2}} \int_{\Omega_{2}}\left\{\Delta[v(x, d)-\widetilde{v}(x, d)]+k_{2}^{2} \widetilde{n}(x)[v(x, d)-\widetilde{v}(x, d)]\right\} \widetilde{v}(x) d x
\end{aligned}
$$




$$
\begin{aligned}
& =-\frac{1}{k_{2}^{2}} \int_{\Omega_{2}}[v(x, d)-\widetilde{v}(x, d)]\left[\Delta \widetilde{v}(x)+k_{2}^{2} \widetilde{n}(x) \widetilde{v}(x)\right] d x \\
& =0 .
\end{aligned}
$$

Then we have to prove that any general case $v(x)$ can be approximated by functions $v(x, d)$ in $L^{2}\left(\Omega_{2}\right)$, where $d \in \mathbb{S}$. If not, that is, the set $\{v(x, d) \mid d \in \mathbb{S}\}$ is not dense in $L^{2}\left(\Omega_{2}\right)$ sense in $\left\{\dot{v}(x) \in C^{2}\left(\overline{\Omega_{2}}\right) \mid \Delta \dot{v}(x)+k_{2}^{2} n(x) \hat{v}(x)=0\right.$ in $\left.\Omega_{2}\right\}$. Then, from the Hahn-Banach theorem, we see that there exists an $f_{3}(x) \in L^{2}\left(\Omega_{2}\right)$ such that

$$
\int_{\Omega_{2}} f_{3}(x) v(x, d) d x=0
$$

for $v(x, d)$ with all $d \in \mathbb{S}$, but for some $\dot{v}(x) \in\left\{\dot{v}(x) \in C^{2}\left(\overline{\Omega_{2}}\right) \mid \Delta \dot{v}(x)+k_{2}^{2} n(x) \dot{v}(x)=0\right.$ in $\left.\Omega_{2}\right\}$,

$$
\int_{\Omega_{2}} f_{3}(x) \dot{v}(x) d x \neq 0
$$

Assume that $\grave{v}(x) \in H^{2}\left(\Omega_{2}\right)$ is a solution to the following interior Neumann problem:

$$
\Delta \grave{v}(x)+k_{2}^{2} n(x) \grave{v}(x)=f_{3}(x) \quad \text { in } \Omega_{2}, \quad \frac{\partial \grave{v}(x)}{\partial v}=0 \quad \text { on } S_{1} .
$$

From (4.31) and (4.33), and by using Green's first theorem, we can see that

$$
\begin{aligned}
0 & =\int_{\Omega_{2}} f_{3}(x) v(x, d) d x=\int_{\Omega_{2}}\left[\Delta \grave{v}(x)+k_{2}^{2} n(x) \grave{v}(x)\right] v(x, d) d x \\
& =\int_{S_{1}} \grave{v}(x) \frac{\partial v(x, d)}{\partial v} d s .
\end{aligned}
$$

From Lemma 8, we know that $\grave{v}(x)=0$ on $S_{1}$. Therefore, by using Green's first theorem, we can see that

$$
\begin{aligned}
\int_{\Omega_{2}} f_{3}(x) \dot{v}(x) d x & =\int_{\Omega_{2}}\left[\Delta \grave{v}(x)+k_{2}^{2} n(x) \grave{v}(x)\right] \hat{v}(x) d x \\
& =\int_{\Omega_{2}}\left[\Delta \dot{v}(x)+k_{2}^{2} n(x) \hat{v}(x)\right] \grave{v}(x) d x=0,
\end{aligned}
$$

which contradicts (4.32). It finishes the proof of the lemma.

So we can obtain our second result as follows.

Theorem 2 Assume that the penetrable interfaces $S_{i}(i=1,2)$ are known and $k_{2}^{2}$ is not a Neumann eigenvalue of $\Delta v(x)+k_{2}^{2} n(x) v(x)=0$ in $\Omega_{2}$, then the refractive index $n(x)$ of the inhomogeneous penetrable obstacle $\Omega_{2}$ can be uniquely determined from the far-field pattern $u^{\infty}(\widehat{x}, d)(\widehat{x}, d \in \mathbb{S})$ for incident plane waves $u^{i}(x)=e^{i k_{1} x \cdot d}$.

Proof By using the completeness result of Lemma 8 and the orthogonality relation of Lemma 9 , we can easily prove the uniqueness result. 


\section{Competing interests}

The authors declare that they have no competing interests.

\section{Authors' contributions}

This paper is the result of joint work of all authors who contributed equally to the final version of this paper. All authors read and approved the final manuscript.

\section{Author details}

'Department of Mathematics, Zhejiang University of Technology, Hangzhou, 310023, China. ${ }^{2}$ Department of Mathematics, Chongqing Normal University, Chongqing, 401331, China.

\section{Acknowledgements}

The first author was supported by National Natural Science Foundation of China under Grant Nos. 11601481 and 11526185. The second author was supported by the National Natural Science Foundation of China under Grant No. 11426052, the Program of Chongqing Education Commission under Grant Nos. KJ1600329 and KJ1400522, and the Program of Chongqing Innovation Team Project in University under Grant No. KJTD201308.

Received: 1 August 2016 Accepted:6 December 2016 Published online: 27 January 2017

\section{References}

1. Hähner, P: A periodic Faddeev-type solution operator. J. Differ. Equ. 128, 300-308 (1996)

2. Nachman, A: Reconstructions from boundary measurements. Ann. Math. 128, 531-576 (1998)

3. Novikov, R: Multidimensional inverse spectral problems for the equation $-\Delta \psi+(v(x)+E u(x)) \psi=0$. Funct. Anal. Appl. 22, 263-272 (1998)

4. Ramm, AG: Recovery of the potential from fixed energy scattering data. Inverse Probl. 4, 877-886 (1988)

5. Ramm, AG: Multidimensional Inverse Scattering Problems. Longman Scientific \& Wiley, New York (1992)

6. Colton, D, Kress, R: Inverse Acoustic and Electromagnetic Scattering Theory, 3rd edn. Springer, Berlin (2012)

7. Isakov, V: Inverse Problems for Partial Differential Equations, 2nd edn. Springer, Berlin (2006)

8. Kirsch, A: An Introduction to the Mathematical Theory of Inverse Problems. Springer, New York (1996)

9. Liu, X, Zhang, B: Direct and inverse obstacle scattering problems in a piecewise homogeneous medium. SIAM J. Appl. Math. 70, 3105-3120 (2010)

10. Liu, X, Zhang, B, Hu, G: Uniqueness in the inverse scattering problem in a piecewise homogeneous medium. Inverse Probl. 26, 015002 (2010)

11. Hähner, P: An uniqueness theorem for an inverse scattering problem in an exterior domain. SIAM J. Math. Anal. 28, 1118-1128 (1998)

12. Athanasiadis, C, Stratis, IG: On some elliptic transmission problems. Ann. Pol. Math. 63, 137-154 (1996)

13. Kirsch, A, Päivärinta, L: On recovering obstacles inside inhomogeneities. Math. Methods Appl. Sci. 21, 619-651 (1998)

14. Liu, X, Zhang, B: Inverse scattering by an inhomogeneous penetrable obstacle in a piecewise homogeneous medium. Acta Math. Sci. 32(4), 1281-1297 (2012)

15. Nachman, A, Päivärinta, L, Teirlilä, A: On imaging obstacle inside inhomogeneous media. J. Funct. Anal. 252, $490-516$ (2007)

16. Yan, G: Inverse scattering by a multilayered obstacle. Comput. Math. Appl. 48, 1801-1810 (2004)

17. Giorgi, G, Brignone, M, Aramini, R, Piana, M: Application of the inhomogeneous Lippmann-Schwinger equation to inverse scattering problems. SIAM J. Appl. Math. 73(1), 212-231 (2013)

18. Qin, Y, Liu, L: Integral equation method for acoustic scattering by an inhomogeneous penetrable obstacle in a stratified medium. Appl. Anal. 93(11), 2402-2412 (2014)

19. Colton, D, Kress, R: Integral Equation Methods in Scattering Theory. Wiley, New York (1983)

20. Kirsh, A, Kress, R: Uniqueness in inverse obstacle scattering. Inverse Probl. 9, 285-299 (1993)

21. Kress, R: Acoustic Scattering: Specific Theoretical Tools. Academic Press, London (2001)

\section{Submit your manuscript to a SpringerOpen ${ }^{\circ}$ journal and benefit from:}

- Convenient online submission

Rigorous peer review

- Immediate publication on acceptance

- Open access: articles freely available online

- High visibility within the field

- Retaining the copyright to your article 